\title{
Formulação de ração para frangos de corte de crescimento lento utilizando valores de energia metabolizável dos ingredientes determinada com linhagens de crescimento lento e rápido
}

\author{
[Feed formulation for slow-growing broilers using metabolizable energy \\ values of food determined with strains of slow-and-fast-growing] \\ F.R. Santos ${ }^{1}$, J.H. Stringhini ${ }^{2}$, C.S. Minafra ${ }^{1}$, R.R. Almeida ${ }^{3}$, P.R. Oliveira ${ }^{4}$, \\ E.F. Duarte ${ }^{3}$, R.B. Silva ${ }^{3}$, M.B. Cafe ${ }^{2}$ \\ ${ }^{1}$ Instituto Federal Goiano - Rio Verde, GO \\ ${ }^{2}$ Escola de Veterinária e Zootecnia - Universidade Federal de Goiás - Goiânia, GO \\ ${ }^{3}$ Aluno de graduação - Instituto Federal Goiano Rio Verde - Rio Verde, GO \\ ${ }^{4}$ Aluno de pós-graduação -Instituto Federal Goiano - Câmpus Rio Verde - Rio Verde, GO
}

\section{RESUMO}

Com este trabalho, objetivou-se avaliar o efeito da formulação de rações para frangos de corte de crescimento lento, com a utilização de valores de energia metabolizável (EMAn) dos alimentos determinados por linhagens de crescimento rápido e lento, sobre o desempenho, características de carcaça, biometria de órgãos digestórios e valores energéticos das rações experimentais. Utilizaram-se 72 pintos de um dia, machos, da linhagem Isa Label, distribuídos em delineamento de blocos ao acaso, com dois tratamentos (energia dos alimentos) e seis repetições de seis animais cada. As médias foram comparadas pelo teste de Tukey a 5\% de probabilidade. Os tratamentos consistiram na formulação de rações baseadas na energia do milho e no farelo de soja determinados com frangos Cobb (EMAn Cobb) e Isa Label (EMAn Isa Label). O experimento foi conduzido na fase inicial, e as aves consumiram as dietas por 28 dias. A formulação da dieta baseada na EMAn Cobb e Isa Label não afetou o desempenho, a biometria de órgãos digestórios e as características de carcaça de aves de crescimento lento. Porém, a dieta formulada com a EMAn Isa Label resultou em melhor conversão calórica e menor deposição de gordura abdominal na carcaça das aves. EMAn da ração semelhante ao nível calculado foi determinada com a formulação baseada na EMAn do milho e do farelo de soja determinada com a linhagem Isa Label $(2.750 \times 2.728 \mathrm{kcal} / \mathrm{kg})$. Os valores de EMAn do milho e do farelo de soja determinados com aves Isa Label não afetam o desempenho dos frangos de crescimento lento, porém resultam em menor gordura abdominal, atendendo aos anseios dos consumidores de aves alternativas.

Palavras-chave: desempenho, gordura abdominal, Isa Label, nutrição, valor energético

\begin{abstract}
We conducted this work aiming to evaluate the effect of using the metabolizable energy of food, determined by different strains (AMEn Cobb and AMEn Isa Label), on diet formulation for slow growing broilers on the performance, carcass characteristics, biometrical morphometry measures of digestive organs and energetic values of the experimental diets. A total of 72 male broiler chickens, Isa Label, were used and the experiment followed a randomized blocks design with two treatments (feedstuff energy), and six replicates of six birds each. The averages were compared by Tukey test at 5\% probability. The treatments consisted in formulating diets using the energy of corn and soybean meal determined by Cobb and Isa Label strains. The experiment was conducted in the starter phase and the birds consumed the diets for 28 days. The diet formulated based on AMEn Cobb or Isa Label did not affect the performance, biometrical and morphometry measures of digestive organs and carcass characteristics. However, the diet formulated according to AMEn Isa Label resulted in better caloric conversion, bioeconomic energetic conversion and lower deposition of abdominal fat. AMEn of the diets similar to those calculated levels were determined with the formulation based on AMEn of corn and soybean meal determined for the
\end{abstract}

Recebido em 1 de fevereiro de 2013

Aceito em 25 de junho de 2014

E-mail: fabiana.santos@ifgoiano.edu.br 
line Isa Label (2.750 x $2.728 \mathrm{kcal} / \mathrm{kg})$. Values of AMEn of corn and soybean meal determined with Isa Label strains not affect the performance of slow growing broilers, but resulted in lower abdominal fat according to the desires of consumers for alternative poultry.

Keywords: carcass yield, energy value, Isa Label, nutrition, performance

\section{INTRODUÇÃo}

Na formulação de rações para frangos de corte, a principal preocupação é fornecer energia e aminoácidos em quantidade adequada para as aves. Para isso, há necessidade de se conhecer o valor energético e a digestibilidade dos alimentos.

No cálculo de rações para aves de crescimento lento, são utilizados valores de energia metabolizável que, de acordo com Rostagno et al. (2011), foram determinados com pintos e galos oriundos de linhagens de crescimento rápido. As aves de crescimento lento possuem características próprias, normalmente, apresenta

m curvas e taxas de crescimento diferentes das linhagens comerciais de corte (Santos et al., 2005). Como são aves com menor taxa de desenvolvimento, as exigências energéticas diferem das exigências dos frangos convencionais (Mendonça et al., 2007). Portanto, é possível que essas linhagens aproveitem os nutrientes dos alimentos de maneira diferente das aves de crescimento rápido.

Até o momento, poucas pesquisas foram realizadas para verificar o aproveitamento nutricional dos alimentos com frangos de crescimento lento e compará-los com aves de crescimento rápido (Wada et al., 2004; Rabello et al., 2012; Santos, 2012; Krás et al., 2013). Embora os resultados dessas pesquisas ainda sejam contraditórios, é comum os autores afirmarem que o aproveitamento nutricional dos alimentos por linhagens com menor taxa de desenvolvimento demonstra valores sub ou superestimados em relação às aves de crescimento rápido.

Dessa forma, a formulação de dietas para frangos de crescimento lento com dados tabelados de energia metabolizável dos alimentos determinadas com linhagens de crescimento rápido pode ser considerada uma extrapolação incorreta, uma vez que as aves com menor taxa de desenvolvimento corporal possuem maior proporção do compartimento gástrico. Segundo Verdal et al. (2010), essa particularidade anatômica pode contribuir para o maior aproveitamento energético dos alimentos, fazendo-se necessário formar um banco de dados com valores nutricionais e energéticos de diversos alimentos para aves de crescimento lento.

Com este trabalho objetivou-se avaliar o efeito da formulação de rações para frangos de corte de crescimento lento, utilizando-se os valores de energia metabolizável dos alimentos determinados com aves da linhagem Cobb 500 e Isa Label.

\section{MATERIAL E MÉTODOS}

Um ensaio de desempenho foi realizado no setor de avicultura do Instituto Federal Goiano, Câmpus Rio Verde - GO. O projeto de pesquisa foi aprovado pelo Comitê de Ética em Pesquisa com animais dessa mesma instituição, sob o protocolo de número 012/2012. Foram utilizados 72 pintos de um dia, machos, da linhagem Isa Label, com peso inicial de 38,5 $\pm 1,98 \mathrm{~g}$. As aves foram alojadas em gaiolas de arame galvanizado, com dimensões de $0,90 \mathrm{~m} \times 0,60 \mathrm{~m} \times 0,40 \mathrm{~m}$, em número de seis aves/gaiola.

O delineamento experimental utilizado foi o de blocos ao acaso, considerando cada andar da bateria um bloco, com dois tratamentos (critérios para formulação) e seis repetições de seis aves cada.

Os tratamentos consistiram na formulação das rações isonutritivas utilizando-se valores de energia metabolizável do milho e do farelo de soja determinados com frangos das linhagens Isa Label e Cobb 500, respectivamente, aves de crescimento lento e rápido: ração formulada com base na EMAn dos alimentos determinada com frangos Cobb (EMAn Cobb); ração formulada com base na EMAn dos alimentos determinada com frangos Isa Label (EMAn Isa Label). 
Os valores de EMAn dos alimentos utilizados na formulação foram determinados por Santos (2012), com frangos de crescimento lento e rápido de 10 a 17 dias de idade. Para a ração EMAn Isa Label, utilizaram-se os valores de 3.157 e $2.363 \mathrm{kcal} / \mathrm{kg}$ para o milho e o farelo de soja, respectivamente. Na formulação da ração EMAn Cobb, foram usados valores iguais a 3.036 e $2.227 \mathrm{kcal} / \mathrm{kg}$, respectivamente, para o milho e o farelo de soja. Para os demais níveis de nutrientes dos alimentos utilizados na ração, assim como para as exigências nutricionais dos animais, foram utilizadas as recomendações propostas por Rostagno et al. (2011), exceto para a exigência de energia metabolizável, a qual utilizou-se o nível proposto por Mendonça et al. (2008) para frangos de corte Isa Label na fase inicial de criação.

Como foram consideradas para a formulação diferentes disponibilidades energéticas dos ingredientes, o inerte (areia lavada) foi adicionado para completar a quantidade de ração sem interferir nos níveis dos nutrientes.

A composição das rações experimentais utilizadas está apresentada na Tab. 1. Água e ração foram fornecidas à vontade em todo o período experimental e os comedouros foram abastecidos com ração duas vezes ao dia, para diminuir o desperdício.

A duração do ensaio foi de 28 dias e foram determinados: o consumo de ração (g por ave), o peso médio (g por ave), o ganho de peso ( $\mathrm{g}$ por ave), a conversão alimentar (g de ração: g de peso) e a eficiência alimentar (\%).

A energia metabolizável aparente (EMA), a aparente corrigida (EMAn) e o coeficiente de metabolização aparente da energia bruta (CMAEB) das rações experimentais foram determinados para se verificar qual critério de formulação, EMAn Cobb ou EMAn Isa Label dos ingredientes, foi condizente com o aproveitamento energético das rações pelos frangos de crescimento lento. Para isso, do $14^{\circ}$ ao $17^{\circ}$ dia, foi realizada a coleta total de excretas de acordo com Sakomura e Rostagno (2007). As análises de matéria seca, nitrogênio e energia bruta foram realizadas segundo Silva e Queiroz (2002). Os valores de EMA e EMAn das rações foram calculados por equações descritas por Matterson et al. (1965). O CMAEB das rações experimentais foi calculado utilizando-se a fórmula: $\mathrm{CMAEB}=(\mathrm{EMAn}$ ingerida$/ \mathrm{EMAn}$ excretada) x 100 .

Tabela 1. Composição centesimal e níveis nutricionais calculados das rações experimentais

\begin{tabular}{|c|c|c|}
\hline Ingredientes & $\begin{array}{l}\text { EMAn } \\
\text { Cobb }\end{array}$ & $\begin{array}{c}\text { EMAn } \\
\text { Label }\end{array}$ \\
\hline Milho & 66,85 & 62,32 \\
\hline Farelo de Soja & 29,01 & 29,93 \\
\hline Fosfato bicálcico & 1,51 & 1,52 \\
\hline Calcário & 0,94 & 0,93 \\
\hline Sal comum & 0,41 & 0,42 \\
\hline Lisina - HCl & 0,34 & 0,38 \\
\hline DL-Metionina & 0,20 & 0,21 \\
\hline L-Treonina & 0,16 & 0,18 \\
\hline Premix mineral $^{1}$ & 0,04 & 0,04 \\
\hline Premix vitamínico ${ }^{2}$ & 0,04 & 0,04 \\
\hline Inerte $^{3}$ & 0,00 & 3,54 \\
\hline Óleo de soja & 0,50 & 0,50 \\
\hline Total & 100,00 & 100,00 \\
\hline
\end{tabular}

\begin{tabular}{|c|c|c|}
\hline & & \\
\hline \multicolumn{3}{|l|}{ Composição calculada } \\
\hline${\text { EMAn }(\mathrm{kcal} / \mathrm{kg})^{4}}^{4}$ & 2.750 & 2.750 \\
\hline Proteína bruta, (\%) & 19,80 & 19,80 \\
\hline Cálcio, (\%) & 0,86 & 0,86 \\
\hline $\mathrm{P}$ disponível, (\%) & 0,38 & 0,38 \\
\hline Lisina total, $(\%)$ & 1,26 & 1,26 \\
\hline Lisina digestível, (\%) & 1,17 & 1,17 \\
\hline Metionina total, (\%) & 0,48 & 0,48 \\
\hline Metionina digestível, (\%) & 0,46 & 0,46 \\
\hline Treonina total, (\%) & 0,86 & 0,86 \\
\hline Treonina digestível, (\%) & 0,77 & 0,77 \\
\hline Sódio, (\%) & 0,21 & 0,21 \\
\hline \multicolumn{3}{|c|}{$\begin{array}{l}{ }^{1} \text { Composição por kg do produto: manganês - } \\
75.000 \mathrm{mg} \text {; ferro - } 20.000 \mathrm{mg} \text {; zinco }-50.000 \mathrm{mg} \text {; cobre - } \\
\text { 4.000mg; cobalto - } 200 \mathrm{mg} \text {; iodo - } 1.500 \mathrm{mg} \text { e veículo } \\
\text { q.s.p. }-1.000 \mathrm{~g} \text {. }\end{array}$} \\
\hline \multicolumn{3}{|c|}{${ }^{2}$ Composição por $\mathrm{kg}$ do produto: ácido fólico - } \\
\hline \multicolumn{3}{|c|}{$5.000 \mathrm{mg} / \mathrm{kg}$; biotina $-60 \mathrm{mg} / \mathrm{kg}$; niacina $-87 \mathrm{~g} / \mathrm{kg}$; vit. } \\
\hline \multicolumn{3}{|c|}{$\begin{array}{l}\mathrm{A}-20.000 .000 \mathrm{UI} / \mathrm{kg} ; \text { vit. } \mathrm{B} 1-3.000 \mathrm{mg} / \mathrm{kg} ; \text { vit. B12 } \\
-28.000 \mathrm{mcg} / \mathrm{kg} \text {; vit. B2 }-12 \mathrm{~g} / \mathrm{kg} \text { : Vvtamina B6 - }\end{array}$} \\
\hline \multicolumn{3}{|c|}{$\begin{array}{l}\text { 40.500UI/kg; vit. K3 - 4.800UI/kg e veículo q.s.p. - } \\
1.000 \mathrm{~g} \text {. }\end{array}$} \\
\hline \multicolumn{3}{|l|}{${ }^{3}$ Areia lavada. } \\
\hline \multicolumn{3}{|c|}{${ }^{4}$ EMAn: EMAn Cobb considerou-se para a formulação } \\
\hline \multicolumn{3}{|c|}{$\begin{array}{l}\text { a EMAn dos ingredientes determinada com aves } \\
\text { Cobb; EMAn Isa Label: considerou-se a EMAn dos } \\
\text { ingredientes determinada com aves Isa Label. }\end{array}$} \\
\hline
\end{tabular}


Ao final do ensaio, foram calculados os consumos de energia metabolizável (kcal por ave), de proteína bruta (g) e conversão calórica (kcal ingerida por $\mathrm{g}$ de ganho de peso), considerando-se os valores energéticos e o teor de proteína determinados em cada ração como referência para o cálculo.

Aos 28 dias, realizou-se a avaliação das características de carcaça: rendimento de carcaça (\%), porcentagem de peito, de coxa + sobrecoxa e de gordura abdominal. Para isso, foram selecionadas duas aves com peso médio próximo ao obtido para parcela, que foram abatidas depois do jejum de 12 horas.

Para realização da biometria, os órgãos do sistema digestório foram medidos e pesados seguindo os passos: comprimento do trato gastrintestinal (TGI) em centímetros (cm), medido desde a inserção do esôfago na orofaringe até a comunicação do intestino grosso com a cloaca; peso em gramas (g) do esôfago mais papo; do proventrículo mais moela (g); do pâncreas (g); do intestino delgado (g); do intestino grosso (g), representado pelo peso dos cecos, cólon e reto; fígado sem a vesícula (g). Com essas medidas, determinou-se o peso relativo (\%) dos órgãos digestivos, calculado em porcentagem do peso vivo da ave.

Os dados foram submetidos à análise de variância por meio do programa SISVAR (Sistema para Análise de Variância, versão 5.0), $\mathrm{e}$ as diferenças entre as médias foram determinadas pelo teste de Tukey a 5\% de probabilidade.

\section{RESULTADOS E DISCUSSÃO}

O maior aproveitamento energético da ração experimental foi determinado pelas aves alimentadas com as dietas formuladas com EMAn Cobb (Tab. 2). Não foi verificado efeito dos tratamentos para o CMAEB.

Tabela 2. Energia metabolizável aparente (EMA), aparente corrigida pelo balanço de nitrogênio (EMAn) e coeficiente de metabolizabilidade da energia bruta (CMEB) das rações experimentais expressos na matéria natural

\begin{tabular}{lcccc}
\hline \multicolumn{1}{c}{ Variável } & EMAn Cobb & EMAn Isa Label & $\mathrm{P}^{1}$ & $\mathrm{CV}^{2}, \%$ \\
\hline EMA $(\mathrm{kcal} / \mathrm{kg})$ & $2.908,83 \mathrm{a}$ & $2.744,47 \mathrm{~b}$ & 0,028 & 3,29 \\
EMAn $(\mathrm{kcal} / \mathrm{kg})$ & $2.894,99 \mathrm{a}$ & $2.728,22 \mathrm{~b}$ & 0,027 & 3,34 \\
CMAEB, $(\%)$ & 76,25 & 74,16 & 0,204 & 3,31 \\
\hline
\end{tabular}

Médias seguidas de letras distintas na linha diferem entre si pelo teste Tukey (5\%).

${ }^{1}$ Valor da probabilidade; ${ }^{2}$ coeficiente de variação.

Segundo Santos (2012), as aves de crescimento lento apresentam maior aproveitamento energético do milho e do farelo de soja em relação à linhagem Cobb. Portanto, embora as rações tenham sido formuladas para serem isoenergéticas, caso a hipótese da melhor metabolização energética dos ingredientes pelas aves de crescimento lento se confirmasse, esperava-se determinar na ração EMAn Cobb valor de energia superior ao calculado. Ao se substituírem, na composição da ração EMAn Cobb, os valores de EMAn do milho e do farelo de soja determinados com a linhagem Isa Label, obtém-se para esta dieta o valor energético calculado igual a $2.870 \mathrm{kcal} / \mathrm{kg}$. Esse valor representa um incremento de $120 \mathrm{kcal}$ no nível energético da ração EMAn Cobb em relação à formulação com os dados energéticos do milho e do farelo de soja determinados com as aves Cobb (2.870 x $2.750 \mathrm{kcal} / \mathrm{kg})$.

Pode-se observar que, neste ensaio, o valor determinado de EMAn Cobb da ração experimental ficou próximo ao esperado (2.894,99 x 2.870kcal/kg) (Tab. 2). As aves de crescimento lento resultaram no aproveitamento energético da ração EMAn Cobb superior ao calculado em 144,99kcal, o que representa um acréscimo de 20,83\% ao incremento esperado.

Um número limitado de pesquisas foi realizado com resultados contraditórios, objetivando-se verificar o aproveitamento nutricional de alimentos com frangos de crescimento lento e compará-los aos níveis encontrados com frangos convencionais. Menores valores de EMAn foram 
verificados para milho, farelo e óleo de soja (Wada et al., 2004) e coprodutos do milho, tais como o gérmen integral de milho, o farelo de glúten 21 e 60\% (Rabello et al., 2012) com aves de crescimento lento em relação aos frangos de crescimento rápido. Por outro lado, maior metabolização da energia, EMAn e digestibilidade da fibra detergente ácido da ração foi verificada por Krás et al. (2013) para frangos Label Rouge aos 41 dias de idade. Santos (2012) também observou, para as aves da linhagem Isa Label, aumento no aproveitamento da energia metabolizável do milho em relação às aves Cobb de 5,75\% para a EMA e CMAEB e 3,44\% para a EMAn. Dessa forma, para uma comparação com maior acurácia, seria necessária a avaliação de outros alimentos com ambas as linhagens, com a finalidade de atestar a possível diferença no aproveitamento nutricional entre elas.

Aos 28 dias de idade, o consumo de ração, o peso médio, o ganho de peso, a conversão e a eficiência alimentar, o consumo de EMAn e o consumo de PB dos frangos de crescimento lento não foram afetados pela formulação da ração com energia metabolizável dos alimentos determinada com frangos Cobb e Isa Label (Tab. $3)$.

Diferente dos resultados deste trabalho, Mendes et al. (2004) afirmaram que o nível energético das rações interfere no resultado de desempenho das aves. Normalmente, o aumento do nível de energia das rações resulta em maior ganho de peso e melhor conversão alimentar.

Tabela 3. Desempenho de frangos de corte alimentados com rações formuladas com energia metabolizável dos alimentos determinada com frangos Cobb (EMAn Cobb) e Isa Label (EMAn Isa Label) aos 28 dias de idade

\begin{tabular}{lcccc}
\multicolumn{1}{c}{ Variáveis } & EMAn Cobb & EMAn Isa Label & $\mathrm{P}^{1}$ & $\mathrm{CV}^{2}, \%$ \\
\hline Consumo de ração (g) & $1.392,41 \mathrm{a}$ & $1.401,18 \mathrm{a}$ & 0,827 & 4,71 \\
Peso médio (g) & $758,34 \mathrm{a}$ & $757,19 \mathrm{a}$ & 0,932 & 2,91 \\
Ganho de peso (g) & $719,28 \mathrm{a}$ & $718,00 \mathrm{a}$ & 0,925 & 3,11 \\
Conversão alimentar g:g & $1,94 \mathrm{a}$ & $1,95 \mathrm{a}$ & 0,726 & 4,12 \\
Eficiência alimentar (\%) & $54,51 \mathrm{a}$ & $51,24 \mathrm{a}$ & 0,787 & 4,27 \\
Consumo de EMAn (kcal/ave) & $4.031,01 \mathrm{a}$ & $3.822,70 \mathrm{a}$ & 0,288 & 6,52 \\
Conversão calórica (kcal:g) & $5,60 \mathrm{~b}$ & $5,31 \mathrm{a}$ & 0,041 & 3,81 \\
Consumo de PB (g/ave) & $279,70 \mathrm{a}$ & $288,10 \mathrm{a}$ & 0,370 & 5,25 \\
\hline
\end{tabular}

Médias seguidas de letras distintas na linha diferem entre si pelo teste de Tukey (5\%).

${ }^{1}$ Valor da probabilidade; ${ }^{2}$ coeficiente de variação.

${ }^{3}$ Calculado de acordo com a EMAn determinada das rações (Tab. 3).

Segundo Sakomura et al. (2004), a melhora que ocorre no ganho de peso e na conversão alimentar quando se eleva a energia metabolizável pode ser atribuída à adição de lipídeos na ração, os quais promovem o incremento da densidade calórica, o efeito extracalórico, que consiste no aumento da disponibilidade dos nutrientes dos ingredientes da ração, e o efeito extrametabólico da gordura, que resulta em melhoria da eficiência energética pelo aumento da energia líquida da ração. Porém, neste ensaio, para conter os efeitos da adição de lipídeos na dieta e verificar apenas o potencial da linhagem sobre a metabolização da energia dos alimentos, os níveis de lipídeos de ambas as dietas experimentais foram controlados. Dessa forma, apesar de se observar uma diferença no conteúdo de energia das rações formuladas com EMAn Cobb e Isa Label de 166,67kcal (Tab. 3), é possível que essa variação na disponibilidade energética, sem os efeitos adicionais da inclusão de lipídeos, não tenha sido suficiente para melhorar o desempenho das aves.

Semelhante a este resultado, a ausência de efeito dos níveis energéticos da ração sobre o desempenho das aves de crescimento lento também foi demonstrada por Moreira et al. (2012), que não verificaram diferença no desempenho de frangos de uma linhagem caipira francês exótico alimentados com dietas com 3.000 e $3.100 \mathrm{kcal} / \mathrm{kg}$ de um a 28 dias. Entretanto, os autores observaram que as aves que consumiram ração com $3.200 \mathrm{kcal} / \mathrm{kg}$ 
apresentaram maior peso médio, ganho de peso diário e melhor conversão alimentar. Porém, diferente dos dados determinados neste trabalho, Mendonça et al. (2008) constataram queda linear do consumo de ração e na conversão alimentar à medida que os níveis de energia metabolizável aumentaram (2.600 a $3.200 \mathrm{kcal})$ em dietas de frangos da linhagem Isa Label aos 21 dias de idade.

Constatou-se melhor conversão calórica pelas aves que consumiram a ração formulada com EMAn do milho e do farelo de soja determinada com a linhagem Isa Label (Tab. 3). Como os critérios de formulação não alteraram o ganho de peso e o consumo de ração, a diferença energética de 166,67kcal, anteriormente citada, entre as rações foi o suficiente para promover piora na conversão calórica, demonstrando que, ao aumentar apenas os níveis energéticos das rações, as aves podem ter perdido a eficiência em converter a energia para ganhar peso, conforme evidenciado por Mendonça et al. (2008).

Não houve diferenças entre os tratamentos para o rendimento de carcaça e partes nobres (Tab. 4). A carcaça das aves que consumiram a ração formulada com EMAn Isa Label apresentou $30,68 \%$ menos gordura abdominal.

Tabela 4. Rendimento de carcaça, partes nobres e gordura abdominal de frangos de corte alimentados com rações formuladas com energia metabolizável dos alimentos determinada com frangos Cobb (EMAn Cobb) e Isa Label (EMAn Isa Label) aos 28 dias de idade

\begin{tabular}{lcccc} 
& EMAn Cobb & EMAn Isa Label & $\mathrm{P}^{1}$ & $\mathrm{CV}^{2}, \%$ \\
\hline Carcaça, (\%) & $63,2 \mathrm{a}$ & $65,08 \mathrm{a}$ & 0,370 & 5,31 \\
Peito, (\%) & $28,19 \mathrm{a}$ & $28,65 \mathrm{a}$ & 0,507 & 3,95 \\
Coxas e sobrecoxas, (\%) & $32,57 \mathrm{a}$ & $30,95 \mathrm{a}$ & 0,151 & 5,21 \\
Gordura abdominal, (\%) & $1,76 \mathrm{~b}$ & $1,22 \mathrm{a}$ & 0,013 & 10,81 \\
\hline
\end{tabular}

Médias seguidas de letras distintas na linha diferem entre si pelo teste de Tukey (5\%).

${ }^{1}$ Valor da probabilidade; ${ }^{2}$ coeficiente de variação.

Considerando-se o teor de proteína bruta de $19,8 \%$ e os valores de EMAn determinados para as rações experimentais (Tab. 2), calculou-se a relação caloria:proteína das rações experimentais igual a 137,8 para a EMAn Isa Label e 146,2 para a EMAn Cobb. De acordo com Nascimento et al. (2004), o consumo de rações com níveis mais elevados de energia sem o adequado equilíbrio de proteína na ração limita o crescimento de tecido magro e, dessa forma, o excedente energético é depositado apenas na forma de gordura, em detrimento do aumento da massa muscular. Segundo Mendonça et al. (2008), a relação caloria:proteína recomendada para machos Isa Label na fase inicial é igual a 128, ou seja, inferior ao observado neste ensaio. Assim, pode-se afirmar que a maior relação caloria:proteína verificada na ração EMAn Cobb é um dos fatores que contribuíram para a deposição de gordura abdominal.

Também, verificou-se que, ao formular rações à base de milho e de farelo de soja, para aves de crescimento lento com níveis energéticos de ingredientes determinados com linhagens de crescimento rápido, é fornecida às aves Isa Label uma maior quantidade de energia. De acordo com Laganá et al. (2005), a deposição de gordura é diretamente proporcional à quantidade de energia disponível para síntese, portanto a energia alimentar em excesso é bem correlacionada à deposição de lipídeos na maioria dos animais.

Assim, embora não haja diferenças estatísticas para consumo de EMAn entre os tratamentos, as aves alimentadas com EMAn Cobb consumiram, no período de 28 dias de criação, em média, $208,31 \mathrm{kcal}$ a mais que as alimentadas com a EMAn Isa Label. Considerando-se que a EMAn Cobb determinada das rações foi igual a 2.894,99 (Tab. 3) e que a exigência para machos Isa Label para essa idade é de $2.750 \mathrm{kcal}$ (Mendonça et al., 2008), observa-se que houve um excedente energético, que provavelmente foi desviado para o metabolismo lipídico, resultando em maior produção de triglicerídeos para deposição nos adipócitos, no caso das aves, principalmente nas gorduras subcutânea e abdominal.

Ressalta-se que o mercado consumidor de aves alternativas tem preferência por carcaças mais 
magras; assim, tecnologias que mantenham as características de carcaças peculiares são de extrema importância para que o produto final atenda aos anseios dos consumidores. Com os resultados obtidos, pode-se afirmar que, ao se formular rações para aves de crescimento lento, é necessário calcular com precisão a quantidade de energia e proteína fornecida, com a finalidade de evitar desbalanceamentos que, de acordo com Nascimento et al. (2004), podem elevar o teor na gordura na carcaça.
Verificou-se maior comprimento do TGI das aves alimentadas com EMAn Cobb (Tab. 5). Os maiores níveis energéticos na ração EMAn Cobb podem ter estimulado o desenvolvimento do comprimento do intestino delgado, uma vez que, logo após a eclosão, a maior parte da demanda de energia e proteína das aves é direcionada para o desenvolvimento do trato digestório, principalmente do intestino (Xavier et al., 2008). Entretanto, não foi observada influência da formulação por EMAn Cobb ou EMAn Isa Label sobre os demais órgãos digestórios de frangos de corte de crescimento lento.

Tabela 5. Biometria de órgãos digestórios de frangos de corte alimentados com rações formuladas com energia metabolizável dos alimentos determinada com frangos Cobb (EMAn Cobb) e Isa Label (EMAn Isa Label) aos 28 dias de idade

\begin{tabular}{lcccc}
\hline \multicolumn{1}{c}{ Variável } & EMAn Cobb & EMAn Isa Label & $\mathrm{P}^{1}$ & $\mathrm{CV}^{2}, \%$ \\
\hline Comprimento do TGI (cm) & $155,33 \mathrm{a}$ & $142,17 \mathrm{~b}$ & 0,020 & 4,53 \\
Esôfago+papo, (\%) & $0,50 \mathrm{a}$ & $0,40^{\mathrm{a}}$ & 0,081 & 15,09 \\
Proventrículo+moela, (\%) & $4,18 \mathrm{a}$ & $4,21 \mathrm{a}$ & 0,897 & 9,45 \\
Intestino delgado, (\%) & $4,12 \mathrm{a}$ & $4,11 \mathrm{a}$ & 0,961 & 10,22 \\
Intestino grosso, (\%) & $1,07 \mathrm{a}$ & $1,01 \mathrm{a}$ & 0,567 & 7,23 \\
Fígado, (\%) & $2,43 \mathrm{a}$ & $2,46 \mathrm{a}$ & 0,788 & 8,77 \\
Pâncreas, (\%) & 0,31a & $0,37 \mathrm{a}$ & 0,077 & 13,10 \\
\hline
\end{tabular}

Médias seguidas de letras distintas na linha diferem entre si pelo teste Tukey (5\%).

${ }^{1}$ Valor da probabilidade; ${ }^{2}$ coeficiente de variação.

A elevação dos níveis energéticos das rações pode estimular o desenvolvimento do TGI, bem como a digestão e a absorção de nutrientes, aumentando também o ganho de peso das aves. Entretanto, neste experimento, não houve diferença entre os tratamentos para o peso corporal das aves Isa Label (Tab. 4). Dessa forma, é possível afirmar que o ganho de massa muscular ocorreu em consonância com o crescimento dos órgãos digestórios, ou seja, a disponibilidade de energia não gerou uma resposta adaptativa no peso dos órgãos do TGI e de suas glândulas anexas.

Semelhantemente aos dados observados neste trabalho, Fagundes (2011) avaliou o efeito de rações com diferentes níveis de energia metabolizável (2900 a 3050kcal $/ \mathrm{kg})$ sobre o desenvolvimento do sistema digestório e a capacidade digestiva em frangos de corte de um a 21 dias de idade e observou que o nível energético não influenciou o peso relativo dos órgãos do TGI. Por outro lado, Ghafari et al. (2008), ao ajustarem os níveis de energia metabolizável aos níveis de aminoácidos totais e digestíveis na ração, verificaram, aos 49 dias de idade, que as aves que receberam dietas com alto nível energético apresentaram menores pesos relativos do TGI.

\section{CONCLUSÕES}

Não há diferença no desempenho, na biometria de órgãos digestórios, no rendimento de carcaça e de partes de aves alimentadas com rações formuladas com energia metabolizável de alimentos determinadas com frangos Cobb e Isa Label. A formulação de rações para aves de crescimento lento com base na EMAn dos ingredientes determinada com frangos Isa Label proporciona às aves melhor conversão calórica e menor deposição de gordura abdominal na carcaça, atendendo aos anseios dos consumidores de aves alternativas.

\section{AGRADECIMENTOS}

À Capes, pelo financiamento da pesquisa por meio do programa DINTER IFGoiano/UFG. 


\section{REFERÊNCIAS}

FAGUNDES, N.S. Desenvolvimento do sistema digestório e da capacidade digestiva de frangos de corte alimentados com diferentes níveis de energia metabolizável. Uberlândia: Universidade Federal de Uberlândia, 2011. 85f. Dissertação (Mestrado em Ciências Veterinárias) - Universidade Federal de Uberlândia, Uberlândia.

GHAFARI, M.; SHIVAZAD, M.; ZAGHARI, M.; SEYFI, E. Determination of the best level of dietary energy with two diet formulation methods based on total and digestible amino acid on broiler diet. Pak. J. Biol. Sci., v.11, p.1461-1466, 2008.

KRÁS, R.V.; KESSLER, A.M.; RIBEIRO, A.M.L. et al. Effect of dietary fiber and genetic strain on the performance and energy balance of broiler chickens. Rev. Bras.Cienc.Avic., v.15, p.15-19, 2013.

LAGANÁ, C.; RIBEIRO, A.M.L.; KESSLER, A.M.; SOUZA, E.N. Influência do nível nutricional da dieta no rendimento de órgãos e gordura abdominal em frangos estressados por calor. Rev. Bras. Saúde Prod. Anim., v.6, p.59-66, 2005.

MATTERSON, L.S.; POTTER, L.M.; STUTZ, M.W.; SINGSEN, E.P. The metabolizable energy of feed ingredients for chickens. University of Connecticut Storrs: Agricultural Experiment Station, 1965, v.11. 11p. (Research Report).

MENDES, A.A.; MOREIRA, J.; OLIVEIRA, E.G. et al. Efeitos da energia da dieta sobre desempenho, rendimento de carcaça e gordura abdominal de frangos de corte. Rev. Bras. Zootec., Viçosa, v.33, p.23002307, 2004.

MENDONÇA, M.O.; SAKOMURA, N.K.; SANTOS, F.R. et al. Níveis de energia metabolizável e relações energia:proteína para aves de corte de crescimento lento criadas em sistema semiconfinado. Acta Sci. Anim. Sci., v.37, p.23-30, 2007.

MENDONÇA, M.O.; SAKOMURA, N.K.; SANTOS, F.R. et al. Níveis de energia metabolizável para machos de corte de crescimento lento criados em semiconfinamento. Rev. Bras. Zootec., v.37, p.14331440, 2008.

MOREIRA, A.S.; SANTOS, M.S.V.; VIEIRA, S.S. et al. Desempenho de frangos caipiras alimentados com rações contendo diferentes níveis de energia metabolizável. Arq. Bras. Med. Vet. Zootec., v.64, p.1009-1016, 2012.
NASCIMENTO, A.H.; SILVA, J.H.V.; ALBINO, L.F.T. et al. Energia Metabolizável e Relação Energia:Proteína Bruta nas Fases Pré-Inicial e Inicial de Frangos de Corte. Rev. Bras. Zootec., v.33, p.911918, 2004.

RABELLO, C.B.V.; SILVA, A.F.; LIMA, S.B.P. et al. Farelo de glúten de milho na alimentação de frangas de corte de crescimento lento. Rev. Bras. Cienc. Agrár., v.7, p.367-37, 2012.

ROSTAGNO, H.S.; ALBINO, L.F.T.; DONZELE, J.L. et al. Tabelas brasileiras para aves e suínos (composição de alimentos e exigências nutricionais). 3.ed. Viçosa: Universidade Federal de Viçosa, 2011. 252p.

SAKOMURA, N.K.; LONGO, F.A.; RABELO, C.B. et al. Efeito dos níveis de energia metabolizável da dieta no desempenho e metabolismo energético de frangos de corte. Rev. Bras. Zootec., v.33, supl. 1, 2004.

SAKOMURA, N.K.; ROSTAGNO, H.S. Métodos de pesquisa em nutrição de monogástricos. Jaboticabal: Funep, 2007. 283p.

SANTOS, F.R. Comparação da eficiência digestiva e metabolismo de nutrientes e de energia entre frangos de crescimento lento e rápido. 2012. 83f. Tese (Doutorado em Ciência Animal). Universidade Federal de Goiás, Faculdade de Medicina Veterinária e Zootecnia, Goiânia.

SANTOS, A.L.; SAKOMURA, N.K.; FREITAS, E.R. et al. Estudo do crescimento, desempenho, rendimento de carcaça e qualidade de carne de três linhagens de frango de corte. Rev. Bras. Zootec., v.34, p.1589-1598, 2005.

SILVA, D.J.; QUEIROZ, A.C. Análise de alimentos (métodos químicos e biológicos). 3.ed. Viçosa, MG: Editora UFV, 2002. 235p.

VERDAL, H.; MIGNON-GRASTEAU, S.; JEULIN, C. et al. Digestive tract measurements and histological adaptation in broiler lines divergently selected for digestive efficiency. Poult. Sci., v.89, p.1955-1961, 2010.

WADA, M.T.; SAKOMURA, N.K.; MENDONÇA, M.O. et al. Determinação dos valores de energia metabolizável de alguns ingredientes utilizados na alimentação de frangos de corte tipo caipira. Rev. Bras. Cienc. Avic., supl. 6, p.59, 2004.

XAVIER, S.A.G.; STRINGHINI, J.H.; BRITO, A.B. et al. Níveis de energia metabolizável em rações iniciais para frangos de corte. Rev. Bras. Zootec., v.37, p.109-115, 2008. 